\title{
Equol effects on AGE skin products, visceral fat, and climacteric
}

symptoms in post-menopausal women

Remi Yoshikata, MD, $\mathrm{PhD}^{1,2}$; Khin Z. Myint, MBBS, MHS'; Hiroaki Ohta, $\mathrm{MD}, \mathrm{PhD}^{3}$; Yoko Ishigaki, $\mathrm{MD}, \mathrm{PhD}^{4}$

${ }^{1}$ Hamasite Clinic, Minato-ku, Tokyo, Japan

${ }^{2}$ Tokyo Midtown Medical Center, Minato-ku, Tokyo, Japan

${ }^{3}$ Sanno Medical Center, Minato-ku, Tokyo, Japan

${ }^{4}$ Sendai Medical Center, Sendai-shi, Miyagi, Japan

Corresponding Author:

Khin Zay Yar Myint, MBBS, MHS

Tokyo Midtown Medical Center, 9-7-1, Akasaka, Minato-ku, Tokyo, 107-6206

Telephone number: +81-3-5413-0083

Fax number: +81-3-5413-1022

E-mail: khinzayarmyint@gmail.com 


\title{
Equol effects on AGE skin products, visceral fat, and climacteric symptoms in post-menopausal women
}

\begin{abstract}
Objective: We investigated the effect of an equol-containing supplement on metabolism and aging, and climacteric symptoms, with respect to internallyproduced equol.
\end{abstract}

Methods: A single center, randomized controlled trial (registration number: UMIN000030975) on 57 post-menopausal Japanese women (mean age: 56 \pm 5.37 years), was conducted. Twenty-seven women received the equol supplement, while the remaining received placebo. Metabolic and aging-related biomarkers were compared before and after the 3-month intervention. Climacteric symptoms were assessed every month using a validated self-administered questionnaire in Japanese post-menopausal women.

Results: Three months post intervention, the treatment group showed significant improvement in climacteric symptoms, when compared to the placebo group ( $81 \%$ vs. $53 \%$ respectively, $\mathrm{p}=0.045$ ). We did not observe any beneficial effect on metabolic and aging-related biomarkers in the intervention group. However, in certain populations significant improvement in skin auto-fluorescence, which is a measurement of AGE skin products, and visceral fat area was observed, especially among equol producers.

Conclusion: Women receiving equol supplement showed improved climacteric symptoms. This study offered a new hypothesis that there may be a synergy between supplemented equol and endogenously-produced equol to improve skin aging and visceral fat in certain populations.

Keywords: equol; advanced glycation end products; visceral fat; climacteric symptoms 


\section{Introduction}

Isoflavones mainly occur as glycosides of glycitein, daidzein, and genistein. In the large intestine, daidzein is converted into equol by the action of the intestinal microbiota. The production of equol from daidzein can be achieved only by a certain type of bacterial species [1]. Moreover, equol metabolism is influenced by the genetic variant [2]. The number of equol producers is lower among people in western countries compared to Asians [3-6], most probably due to the influence of dietary habits. However, the younger generations in Asian countries were found to possess lower number of equol producers, which might be due to dietary changes and the widespread use of antibiotics $[7,8]$.

Equol is chemically similar to estrogen. Therefore, its estrogenic actions are thought to be exerted through the estrogen receptors, alpha and beta $[9,10]$, or through the $\mathrm{G}$ protein-coupled estrogen receptors $[11,12]$. The benefits of being equol producers range from relieved climacteric symptoms $[13,14]$, to prevention of bone density loss [15], and reduced risk of lifestyle-related diseases and cancers [16-20]. After the commercial availability of equol supplements, some of the above benefits had been tested with the use of equol supplement [21-25].

In recent years, advanced glycation end products (AGEs) have been regarded as one of the contributing factors for lifestyle-related diseases and aging. With regards to menopause, AGEs may enhance ovarian aging by increasing oxidative stress [30], initiate bone remodeling, and increase the risk of osteoporosis in post-menopausal women [31], although there was no report on its effects on climacteric symptoms. AGEs cause stiffness and loss of elasticity through crosslinking of tissue protein such as collagen and elastin in the vessels and skin cells [26]. Therefore, the level of AGEs in 
the skin can be determined non-invasively by using AGE reader through measuring the skin autofluorescence or SAF [27-29].

Estradiol and isoflavones have been reported to inhibit the production of AGEs [32,33]. As a potent isoflavone derivative and an estrogenic agent, equol is expected to exert similar effects on AGEs. However, to our knowledge, there have been no equolbased trials to study their effect on AGEs. Similarly, there have been no clinical trials on the effect of equol supplements on visceral fat. Although equol producers have been reported to be associated with reduced body and visceral fat levels [16,34], literature regarding the action of equol supplement with respect to endogenously-produced equol is trivial.

In this study, we aimed to investigate the effect of equol supplementation on AGEs and visceral fat areas, in addition to other metabolic and aging-related markers. We also assessed the efficacy of the equol supplement at management of climacteric symptoms, in both equol producers and non-producers.

\section{Methods}

\section{Study population}

A randomized controlled trial was conducted for 3 months, at the Sendai Medical Center in Sendai City, Miyagi Prefecture, located in North Eastern Japan. Postmenopausal women who underwent medical check-up in January 2018 were recruited to the study. Among the 85 women enrolled in the study, 63 were selected based on the inclusion and exclusion criteria as follows.

Inclusion criteria: (1) Postmenopausal women (natural absence of menstruation over at least 12 months since the last menstrual period or a bilateral oophorectomy procedure performed in a woman (surgical menopause); (2) Those who could visit the clinic every 
month for interviews or investigations during the study period; (3) Those who could remain compliant with the daily supplementation regimen and record daily notes throughout the study period comprising 12 weeks.

Exclusion criteria: (1) Those with history of allergy to soy foods, dairy products, or Brewer's yeast; (2) Those who reported intake of medications or functional foods that could affect our study results; (3) Those administered hormone therapy or medications that could affect blood hormone levels; (4) Those considered ineligible by the investigators.

The details of the study were explained to these women, and the first 60 women to give consent to participate in the study were selected. They were assigned into two groups. One group received a $10 \mathrm{mg}$ equol supplement and lactobionic acid daily, while another group received placebo. Each patient had a regular follow-up every month, for 3 months. In the equol-supplementation group, one woman dropped out and two women reported that they had occasionally used the equol supplement before the intervention. Consequently, 57 women (48 to 69 years of age) were eventually included for analysis in the study (Figure 1).

\section{Study treatments}

Twenty seven women belonging to the equol intervention group received $10 \mathrm{mg}$ of oral equol-containing supplement per day, composed of $98 \%$ S-equol, $2 \%$ daidzein, $0.2 \%$ glycitein, and $0.1 \%$ genistein extracted from fermented soy beans (product name: FlavoCel EQ-5, Daicel Corporation, Tokyo, Japan).

\section{Determination of equol-producer status}

Prior to the intervention, early morning urine samples were collected from all 
participants. Urinary equol was measured using an immunochromatographic strip (Soy Check, Healthcare Systems Co., Ltd), as described in a previous study [15]. Individuals were considered as equol-producers if their urinary equol level was higher than $1.0 \mu \mathrm{M}$, as described in previous studies $[3,4]$.

\section{Measures}

Body height and weight were measured using a height weight scale (A \& D Company Limited, Tokyo, Japan). Overnight fasting blood samples were obtained to determine the levels of triglycerides (TG), total cholesterol (TC), low density lipoprotein (LDL) cholesterol, high density lipoprotein (HDL) cholesterol, hemoglobin A1c (HbA1c), uric acid (UA), intact parathyroid hormone (PTH), and 25-hydroxy vitamin D. To assess the degree of arterial stiffness (arteriosclerosis), brachial-ankle pulse wave velocity (baPWV) was measured using vascular ultrasound (Fukuda Denshi, Tokyo, Japan). Visceral fat area was measured using computed tomography (CT). The level of AGEs was determined by measuring the skin autofluorescence on the volar side of the forearm using an AGE reader ((DiagnOptics, Groningen, Netherlands) as described previously [26]. Autofluorescence was defined as the average fluorescence per nm over the entire emission spectrum (420-600 nm) as ratio of the average fluorescence per nm over the 300-420-nm range [26]. Age-adjusted SAF levels ( $z$ scores) were calculated for each woman, based on the total population. The above measurement of parameters was performed at the baseline and 3-months post intervention.

\section{Secondary outcomemeasures}

Self-administered questionnaires were used for the assessment of climacteric symptoms, using the Climacteric Scale developed by the Japan Society of Obstetrics and 
Gynecology (table 1). The questionnaire contained 21 items scored on a 4-point scale $($ Never $=0$, mild $=1$, moderate $=2$, severe $=3)$. The total score represented the overall severity of the symptoms. These questionnaires were administered during the monthly follow-ups, for 3 months. Starting from the first month of the intervention, the overall improvement in symptoms was also assessed using the following four responses: 'a lot', 'somewhat', 'no change', and 'worse'. This technique was validated and widely used in the Japanese population.

\section{Treatment adherence and monitoring of adverse effects}

During the monthly follow-ups, the staff interviewed the adherence to equol supplement such as frequency and dose, as well as clinical signs and symptoms of adverse effects in order to ensure compliance as well as make timely decision on cessation of the supplement.

\section{Statistical analyses}

All statistical analyses were performed using the IBM SPSS 19 statistical software (IBM Japan, Minato-ku, Tokyo, Japan). The Wilcoxon sign rank test was used to compare the changes with respect to the baseline, in each group, and Mann-Whitney test was used to compare the differences between the intervention and control groups. Additionally, we assessed the change in results with respect to equol exposure by categorizing the groups into four categories: 1) exposure to both intrinsic and extrinsic equol, i.e., equol producers consuming equol supplement, 2) exclusively extrinsic equol exposure, i.e., equol non-producers consuming equol supplement, 3) exclusively intrinsic equol exposure, i.e., equol producers without equol supplement, and 4) no equol exposure, i.e., equol non-producers without supplement. The extended Mantel- 
Haenszel chi square for linear trend was used to examine the association between postintervention improvement (dependent variable) and equol exposure (independent variable).

Changes in the severity (total score) of climacteric symptoms over time, i.e., at baseline, 1 month, 2 months and 3 months post equol intervention., were analyzed using a twoway repeated measure analysis of variance (ANOVA). Analysis of the studentized residuals showed that there was normality, as assessed by the Shapiro-Wilk test of normality and no outliers, as assessed by no studentized residuals greater than \pm 3 standard deviations. There was sphericity for the interaction term, as assessed by Mauchly's test of sphericity $(\mathrm{p}>.05)$. Also, post hoc pairwise comparisons between equol intervention and control groups as well as between equol producers and nonproducers were conducted using the Bonferroni correction. Improvement in climacteric symptoms, in the control and equol intervention groups, were compared using the chisquare test and the Fisher exact test for proportions. All tests were two-sided, and the statistical significance was set to $\mathrm{p}<0.05$.

\section{Results}

\section{Characteristics of participants}

The baseline characteristics of the equol intervention group and the control group showed no statistically significant difference, except for drinking habits and supplement use (Table 2). Among the 57 women analyzed, 13 were equol producers (22.8\%).

\section{Changes after 3 months}

Both groups exhibited a significant decrease in the levels of HDL cholesterol and 25- 
$\mathrm{OH}$ vitamin $\mathrm{D}$, and increase in the level of PTH. In the equol intervention group, a significant reduction was observed in the levels of LDL and total cholesterol. In the control group, the visceral fat level was significantly reduced after 3 months. Although such changes were observed at baseline and after 3 months in both groups, there was no significant difference between the two groups with respect to these changes. Therefore, the proportion of women in the two groups displaying changes from baseline, were compared. Post 3 months, more women in the equol intervention group were found to have either improved or controlled baPWV (within normal limits), compared to those in the control group $(26.7 \%$ vs. $3.7 \%, \mathrm{p}=0.042)$.

\section{Changes with regards to equol exposure categories on ad-hoc analysis}

Initially, no significant difference was observed among the four equol exposure categories: 1) both intrinsic and extrinsic equol exposure, 2) exclusively extrinsic equol exposure, 3) exclusively intrinsic equol exposure, and 4) no equol exposure. However, in the subgroup analysis, the extended Mantel-Haenszel chi square for linear trend showed significant linear trends of improvement in visceral fat area and skin autofluorescence on exposure to equol (table 3).

Figure 2A shows the proportion of women with improved visceral fat area post 3 months, excluding those women on regular exercise, and those consuming lipid lowering medications $(n=39)$. Women with a habit of exercising regularly were excluded from the analysis because regular exercise is the most effective strategy for reducing visceral fat [35]. Visceral fat areas were found to be increased in 6 out of 14 people with no equol exposure, 1 out of 7 people in the exclusively intrinsic exposure group (14.3\%), 1 out of 15 people in the exclusively extrinsic exposure group (13.3\%), and nobody in the intrinsic and extrinsic exposure group (0\%). The extended Mantel- 
Haenszel chi square for linear trend showed significant linear trends $(\mathrm{p}=0.023)$ of improvement in visceral fat area in response to equol exposure.

Figure $2 \mathrm{~B}$ shows the proportion of people, among 50 women, with improved skin auto-fluorescence, post 3 months, measured using AGE reader, excluding those women with current history of medications like lipid lowering agents and anti-diabetics. Women consuming the aforementioned drugs were excluded because these medications could affect the results of skin auto-fluorescence [36,37]. Skin auto-fluorescence was found to be improved in 3 out of 15 people with no equol exposure (16.7\%), in 7 out of 13 people in the exclusively intrinsic exposure group (35\%), in 3 out of 5 people in the exclusively extrinsic exposure group (37.5\%), and in 3 out of 4 people in the group with both intrinsic and extrinsic exposure (75\%). The extended Mantel-Haenszel chi square for linear trend showed significant linear trends $(\mathrm{p}=0.044)$ in improvement of skin autofluorescence in response to equol supplementation.

\section{Changes in climacteric symptom scores}

The two-way repeated measure ANOVA indicated significant time effect ( $F$ $(3,159)=4.055, \mathrm{p}=0.009)$ and group $\mathrm{x}$ time interaction $(\mathrm{F}(3,159)=3.531, \mathrm{p}=0.017)$, suggesting that the climacteric symptoms improved during the study period in the equol intervention group, and that the group effect varied with time. A post hoc pairwise comparison using the Bonferroni correction showed a decrease in total climacteric symptom scores between the initial assessment and follow-up assessment at 2 months after intervention (16.44 vs 12.22 , respectively), which was statistically significant ( $\mathrm{p}=$ 0.014). Furthermore, the decrease in total climacteric symptom scores did reach significance when comparing the initial assessment to a follow-up assessment taken 3 months after the intervention $(16.44$ vs $11.33, \mathrm{p}=.002)$. Therefore, we can conclude 
that the results for the ANOVA indicated that the difference in climacteric symptom improvement in the equol intervention group was more pronounced with time.

However, there was no interaction between the groups with respect to time and equol producer status, indicating that equol supplementation might be beneficial regardless of the equol producer status (Figure 3A).

\section{Changes in the self-reported overall improvement of symptom scores}

The proportion of women who reported overall improvement of symptoms in the equol supplement group was $56 \%$, as compared to $40 \%$ in control group 1 month after the intervention. A marginal increase in this proportion was observed in the control group at 2 months post intervention (53\%), but no change was seen at 3 months. However, these proportions significantly increased in the equol intervention group, especially at 3 months post intervention, where $81 \%$ of the women experienced improvement in climacteric symptoms (Figure 3B).

\section{Discussion}

In this study, only $\sim 22.8 \%$ of the population were equol producers, which is lower than that previously reported $[6,15]$. Gut microbiota gets established immediately after birth [38]. Consequently, it gets shaped according to the individual dietary and lifestyle habits. Our previous study showed that equol production was associated with the type of diet as well as with lifestyle habits [39]. Therefore, individual differences in composition of gut microbiota might be responsible for different results reported in different studies. In recent years, microorganisms that can produce equol have been identified more. Using genetic engineering techniques, these microorganisms could be used for the formulation of functional foods. 
A significant decrease in LDL level was observed in the equol intervention group, which in turn might be responsible for the lower total cholesterol level. Furthermore, a significant reduction in vitamin D, HDL cholesterol and increased PTH levels were noted in both groups. The reason for reduction in the vitamin D level might be due to the reduced production of vitamin $\mathrm{D}$ as exposure to ultraviolent light was reduced during the winter in North Eastern Japan when this study was conducted. As vitamin D level is inversely associated with PTH level, PTH level might be increased as a consequence. In some studies, the HDL level was found to be positively associated with Vitamin D level, in post-menopausal women [40, 41]. Therefore, the fall in the vitamin D level might be responsible for the fall in HDL cholesterol level in both groups. Although the exact mechanism for such association has not been fully elucidated, further studies should be warranted to explore the role of vitamin D in preventing cardiovascular diseases.

The above findings are contradictory to the results obtained in previous studies of post-menopausal women administered with equol supplementation for one year $[23,25]$. One study found that equol supplementation helped to reduce bone mineral density [23]. Another study found improvements in cardiovascular- and bone densityrelated biomarkers like arterial stiffness, triglycerides, urinary NTX, and intact PTH levels [25]. The discrepancy in the results might be due to the fact that the current study was conducted as a short-term intervention among relatively healthy individuals, while previous studies were long-term and were conducted among high risk individuals with pre-existing morbidities. Concordantly, in the current study, we found that arterial stiffness - measured in terms of baPWV level—was improved in women with high baseline baPWV values. 
With respect to visceral fat area, there have been no randomized controlled trials on the effects of equol supplementation. However, one study has reported an association between reduced visceral fat levels and equol producer status [16]. The potential mechanism of action of equol might be due to its estrogenic property. It acts by modulating body fat deposition [42], or by directly acting on the genes involved in energy metabolism [34]. In this study, it was found that greater exposure to equol was less likely to be associated with unhealthy visceral fat levels in postmenopausal women without regular exercise habits, at 3 months post intervention. At least one kind of exposure to equol, either intrinsically via equol production, or extrinsically via equol supplementation, might aid in preventing the worsening of visceral fat levels in postmenopausal women. Possessing both exposures, that is, an equol producer consuming an equol supplement, could be the most effective mechanism for maintaining visceral fat levels. Consequently, it is probable that equol could help reduce central-obesity related diseases in post-menopausal women who could not exercise regularly due to physical morbidities.

In recent years, AGEs have been considered to play an important role in aging. The accumulation of AGEs in the skin can be determined through measuring the skin autofluorescence or SAF, and values are age-adjusted [27-29]. As AGE production requires estrogen, the estrogen level post menopause might affect the AGEs [32, 43]. The effects of isoflavones on AGEs, especially the inhibition of AGE formation, have been reported [33]. Isoflavones act via 3 mechanisms: 1) prevention of Maillard reaction, i.e., prevention of sugar-protein compound formation; 2) inhibition of the oxidation of sugar, fat and amino acids, thereby preventing the formation of carbonyl group; and 3) prevention of the conversion of Schiff base into carbonyl group. As equol is a metabolite of isoflavones, it might exert similar effects as those of isoflavones. 
Moreover, as equol exerts anti-aging and anti-arteriosclerosis effects on the skin [44], the skin auto-fluorescence values could also be affected.

With respect to the improvement in climacteric symptoms, both equol producers as well as non-producers showed similar levels of improvement in the treatment group. Previous studies of equol efficacy on climacteric symptoms had focused on equol nonproducers. In this study, we found that the equol supplementation further improved the climacteric symptoms in equol producers.

Despite the many positive findings, our study had some limitations. Firstly, it was a small scale, single center study, which limited the generalizability. Secondly, we randomly assigned the intervention and control groups, without considering the equol producer status. Although there is no statistically significant difference between the two groups with respect to their equol producer status, it would have been better if we had assigned equal proportions of equol producers and non-producers to both groups. Thirdly, although we assessed food habits at the baseline, we could not assess the changes in food habits after 3 months. Therefore, the type of diet and the amount of isoflavone intake might affect the outcomes $[45,46]$. However, the effect of diet is considered trivial as diet is not the sole determinant of equol production and metabolism $[1,2,47]$. Furthermore, it is not clear if the non-producer status can be converted to producer status without isoflavone intervention within three months, as previous studies have shown that equol production phenotype is relatively stable without dietary intervention [48-49].

Another important limitation of this study was that we were unable to calculate the sample size statistically since we could not find any previous clinical trial on the effects of equol or isoflavone on AGEs or visceral fat to use as a reference for sample size calculation. Therefore, we applied general rule of thumb for sample size 
determination for this study, which is 30 post-menopausal women in each group. In addition, we found out that there may be dose-response relationship depending on individual equol producing ability only after analyzing the data. We were also unaware that exercise or certain medications might have significant effect on AGEs or visceral fat before the study due to insufficient literature review. These factors should have been considered in calculating the sample size before starting the study.

Despite the aforementioned limitations, this is the first study to report the effect of equol supplementation on visceral fat area and AGEs. It also provided a new insight that the action of equol possesses a dose-response relationship, depending on the inherent equol production ability of the population. Greater equol exposure might be more beneficial in correcting visceral adiposity in people without regular exercise, and in protecting against aging. Moreover, since the equol producing ability of an individual was found to be associated with microbial diversity [39], the gut environment might favor the absorption of the equol supplement.

Furthermore, we found that equol supplementation could be beneficial to both equol producers and non-producers for relieving climacteric symptoms. Equol producers tend to have lower severity of climacteric symptoms, with improvement in symptoms associated with the consumption of an equol supplement. Most of the previous studies focused on the benefits of equol in non-equol producer women. Similar to our previous prospective study on postmenopausal women using equol supplement for a year [25], this study also indicated the beneficial effect of equol supplementation on the relief of climacteric symptoms in equol producer women. 


\section{Conclusion}

From this study, we found that equol supplementation had the potential to improve visceral fat area and advanced glycation end product production in certain postmenopausal women. The benefits of equol supplementation might be enhanced in women with inherent equol producing ability. Additionally, equol supplementation led to an overall improvement in climacteric symptoms, regardless of the equol producer status. Consequently, equol might be a potential alternative to HRT for relieving climacteric symptoms. Moreover, it can also be used for the prevention of lifestylerelated diseases, and for aging care in post-menopausal women. However, cautions should be applied since these preliminary findings warrant further investigation to confirm the obtained results.

\section{Declarations}

\section{Ethics approval and consent to participate}

The study was conducted in compliance with the Declaration of Helsinki, and approved by the Institutional Review Board of the Medical Corporation of Shinkokai. All participants provided written informed consents for participating in the study. This study was registered with the University Hospital Medical Information Network (UMIN) Clinical Trial Registry (trial registration number: UMIN000030975).

\section{Consent for publication}

Not applicable

\section{Availability of data and materials}

The datasets used and/or analysed during the current study are available from the corresponding author on reasonable request. 
Competing interests

Advanced Medical Care Inc. provides administrative support to Tokyo Midtown Medical Center.

\section{Funding}

We would like to express our gratitude to the Advanced Medical Care Inc. for providing financial support for this research.

\section{Authors' contributions}

Dr. Remi Yoshikata is the principal investigator, who conceptualized or designed the entire study; drafted and revised the manuscript.

Khin Zay Yar Myint (ORCID: 0000-0001-5553-1245) is the corresponding author, who has conducted data analysis and interpretation; and involved in revising the manuscript.

Hiroaki Ohta involves in revising the work critically for important intellectual content regarding equol research as an academic advisor; and final approval of the version to be submitted.

Yoko Ishigaki is the co-investigator at the Sendai Medical Center, who has contributed to conceptualization, and implementation of the study.

\section{Acknowledgements}

We would like to acknowledge all the women who willingly came forward to participate in the study. 


\section{References:}

1. Rafii F. The Role of Colonic Bacteria in the Metabolism of the Natural Isoflavone Daidzin to Equol. Metabolites 2015; 5(1):56-73.

2. Van de Merwe JP, Stegeman JH, Hazenberg MP. The resident faecal flora is determined by genetic characteristics of the host. Implications for Crohn's disease? Antonie Van Leeuwenhoek 1983; 49 (2):119-12.

3. Lampe JW, Karr SC, Hutchins AM, et al. Urinary Equol Excretion with a Soy Challenge: Influence of Habitual Diet. Proc Soc Exp Biol Med 1998; 217: 335-339.

4. Rowland IR, Wiseman H, Sanders TAB, et al. Interindividual variation in metabolism of soy isoflavones and lignans: Influence of habitual diet on equol production by the gut microflora nutrition and cancer. Nutr Cancer 2000; 36: 27-32.

5. Atkinson C, Frankenfield CL, Lampe JW. Gut bacterial metabolism of the soy isoflavone daidzein: exploring the relevance to human health. Exp Biol Med 2005; 230: 155-170.

6. Akaza H, Miyanaga N, Takashima N, et al. Comparisons of percent equol producers between prostate cancer patients and controls: case-controlled studies of isoflavones in Japanese, Korean and American residents. Jpn J Clin Oncol 2004; 34: 86-9.

7. Van der Velpen V, Hollman PC, van Nielen M, et al. Large inter-individual variation in isoflavone plasma concentration limits use of isoflavone intake data for risk assessment. Eur J Clin Nutr 2014; 68: 1141-7.

8. Franke AA, Lai JF, Halm BM, et al. Equol production changes over time in postmenopausal women. J Nutr Biochem 2012; 23: 573-9.

9. Klinge CM. Estrogen receptor interaction with estrogen response elements. Nucleic Acids Res 2001; 29:2905-2919.

10. Marino M, Galluzzo P, Ascenzi P. Estrogen Signaling Multiple Pathways to Impact Gene Transcription. Curr Genomics 2006; 7(8):497-508. 
11. Nilsson S, Mäkelä S, Treuter E, et al. Mechanisms of estrogen action. Physiol Rev 2001; 81:1535-1565.

12. Heldring N, Pike A, Andersson S, et al. Estrogen receptors: how do they signal and what are their targets. Physiol Rev 2007; 87, 905-931.

13. Uesugi S, Watanabe S, Ishiwata N, et al. Effects of isoflavone supplements on bone metabolic markers and climacteric symptoms in Japanese women. Biofactors $2004 ; 22: 221-8$.

14. North American Menopause Society The role of soy isoflavones in menopausal health: report of The North American Menopause Society/Wulf H. Utian Translational Science Symposium in Chicago, IL (October 2010) Menopause. 2011;18(7):732-53.

15. Wu J, Oka J, Higuchi M, et al. Cooperative effects of isoflavones and exercise on bone and lipid metabolism in postmenopausal Japanese women: a randomized placebocontrolled trial. Metabolism 2006; 55:423-33.

16. Yoshikata R, Myint KZ, Ohta H. Relationship between equol producer status and metabolic parameters in 743 Japanese women. Menopause 2017; 24(2): 216-22.

17. Zheng W, Dai Q, Custer LJ, et al. Urinary excretion of isoflavonoids and the risk of breast cancer. Cancer Epidemiol Biomarkers Prev 1999; 8:35-40.

18. Ingram D, Sanders K, Kolybaba M, Lopez D. Case-control study of phytooestrogens and breast cancer. Lancet 1997; 350:990-4.

19. Akaza H, Miyanaga N, Takashima N, et al. Is daidzein non-metabolizer a high risk for prostate cancer? A case-controlled study of serum soybean isoflavone concentration. Jpn J Clin Oncol 2002; 32:296-300.

20. Ozasa K, Nakao M, Watanabe Y, et al. Serum phytoestrogens and prostate cancer risk in a nested case-control study among Japanese men. Cancer Sci 2004; 95:65-71.

21. Ishiwata N, Melby MK, Mizuno S, et al. New equol supplement for relieving menopausal symptoms: randomized, placebo-controlled trial of Japanese women. Menopause 2009; 16: 141-148. 
22. Aso T. Equol improves menopausal symptoms in Japanese women. J Nutr 2010; 140: 1386S-9S.

23. Tousen Y, Ezaki J, Fujii Y, et al. Natural S-equol decreases bone resorption in postmenopausal, non-equol-producing Japanese women: A pilot randomized, placebocontrolled trial. Menopause. 2011;18:563-574.

24. Hazim S, Curtis PJ, Schär MY, et al. Acute benefits of the microbial-derived isoflavone metabolite equol on arterial stiffness in men prospectively recruited according to equol producer phenotype: A double-blind randomized controlled trial. Am. J. Clin. Nutr. 2016;103:694-702.

25. Yoshikata R, Myint KZ, Ohta H. Effects of equol supplement on bone and cardiovascular parameters in middle-aged Japanese women: Prospective observational study. JACM 2018; 24(7):701-708

26. Singh R, Barden A, Mori T, Beilin L. Advanced glycation end-products: a review. Diabetologia. 2001;44(2):129-46.

27. Meerwaldt R, Graaff R, Oomen PHN, et al. Simple non-invasive assessment of advanced glycation endproduct accumulation. Diabetologia 2004; 47: 1324-30.

28. Bos DC, de Ranitz-Greven WL, de Valk HW. Advanced glycation end products, measured as skin autofluorescence and diabetes complications: a systematic review. Diabetes Technol Ther 2011; 13: 773-9.

29. Yamagishi S, Fukami K, Matsui T. Evaluation of tissue accumulation levels of advanced glycation end products by skin autofluorescence: a novel marker of vascular complications in high-risk patients for cardiovascular disease. Int J Cardiol 2015; 185: 263-8.

30. Pertynska-Marczewska M, Diamanti-Kandarakis E. Aging ovary and the role for advanced glycation end products, Menopause: March 2017 - Volume 24 - Issue 3 - p 345-351 
31. Yang DH, Chiang TI, Chang IC, Lin FH, Wei CC, Cheng YW. Increased levels of circulating advanced glycation end-products in menopausal women with osteoporosis. Int J Med Sci. 2014;11(5):453-460

32. Jackson S, James M, Abrams P. The effect of oestradiol on vaginal collagen metabolism in postmenopausal women with genuine stress incontinence. BJOG. 2002;109:339-344.

33. Silvan JM, Srey C, Ames JM, et al : Glycation is regulated by isoflavones. Food Funct. $2014 ; 5(9)$ : 2036-42

34. van der Velpen V, Geelen A, Hollman PC, et al. Isoflavone supplement composition and equol producer status affect gene expression in adipose tissue: a double-blind, randomized, placebo-controlled crossover trial in postmenopausal women. Am J Clin Nutr 2014; 100: 1269-1277.

35. Rao S, Pandey A, Garg S, et al. Effect of Exercise and Pharmacological Interventions on Visceral Adiposity: A Systematic Review and Meta-analysis of Longterm Randomized Controlled Trials.Mayo Clin Proc 2019;94(2):211-224.

36. Nenna A, Nappi F, Avtaar Singh SS, et al. Pharmacologic Approaches Against Advanced Glycation End Products (AGEs) in Diabetic Cardiovascular Disease. Res Cardiovasc Med. 2015;4(2):e26949.

37. Mirhashemi SM, Rahimi F, Soleimani A, et al. Effects of Omega-3 Fatty Acid Supplementation on Inflammatory Cytokines and Advanced Glycation End Products in Patients With Diabetic Nephropathy A Randomized Controlled Trial. Iranian Journal of Kidney Diseases. 2016;10:197-204.

38. Mändar R, Mikelsaar M. Transmission of mother's microflora to the newborn at birth. Biol Neonate 1996; 69: 30-35.

39. Yoshikata R, Myint KZ, Ohta H, et al. Interrelationship between diet, lifestyle habits, gut microflora, and the equol-producer phenotype : Baseline findings from a placebo-controlled intervention trial. Menopause 2019; 26(3): 273-285 
40. Chon SJ, Yun BH, Jung YS, Cho SH, Choi YS, Kim SY, et al. (2014) Association between Vitamin D Status and Risk of Metabolic Syndrome among Korean Postmenopausal Women. PLoS ONE 9(2): e89721.

41. Schnatz PF, Jiang X, Vila-Wright S, et al. Calcium/vitamin D supplementation, serum 25-hydroxyvitamin D concentrations, and cholesterol profiles in the Women's Health Initiative calcium/vitamin D randomized trial. Menopause. 2014;21(8):823 833.

42. Toth MJ, Tchernof A, Sites CK, et al. Effect of menopausal status on body composition and abdominal fat distribution. Int J Obes Relat Metab Disord 2000; 24: 226-231.

43. Pertynska-Marczewska M, Merhi Z. Relationship of advanced glycation end products with cardiovascular disease in menopausal women. Reprod Sci. 2015;22:774782.

44. Lephart ED. Skin aging and oxidative stress: Equol's anti-aging effects via biochemical and molecular mechanisms. Ageing Res Rev 2016; 11 (31): 36-54.

45. Davinelli S, Scapagnini G, Marzatico F, et al. Influence of equol and resveratrol supplementation on health-related quality of life in menopausal women: A randomized, placebo-controlled study. Maturitas 2017;96 (2):77-83.

46. Kruger MC, Middlemiss C, Katsumata S, et al. The effects of green kiwifruit combined with isoflavones on equol production, bone turnover and gut microflora in healthy postmenopausal women. Asia Pac J Clin Nutr 2018;27(2):347.

47. Miura A, Sugiyama C, Sakakibara H, et al. Bioavailability of isoflavones from soy products in equol producers and non-producers in Japanese women. J Nutr Intermed Metab 2016; 6: 41-47.

48. Védrine N, Mathey J, Morand C, et al. One-month exposure to soy isoflavones did not induce the ability to produce equol in postmenopausal women. Eur. J. Clin Nutr 2006; 60:1039-1045. 
49. Mathey J, Lamothe V, Coxam V, et al. Concentrations of isoflavones in plasma and urine of post-menopausal women chronically ingesting high quantities of soy isoflavones. J. Pharm. Biomed Anal 2006; 41:957-965.

50. Frankenfeld CL, Atkinson C, Thomas WK, et al. High concordance of daidzeinmetabolizing phenotypes in individuals measured 1 to 3 years apart. Br. J. Nutr. 2005; 94:873-876. 
Table 1. Climacteric scale items by the Japan Society of Obstetrics and Gynecology Table 2. Basic characteristics of equol intervention group and control group

Table 3. Changes with regards to equol exposure categories on ad-hoc analysis for visceral fat and advanced glycation end products

Figure 1. Flow diagram of the study

Figure 2. Effects of intrinsic and extrinsic equol exposure after 3 months

Figure 3. Effect of equol on climacteric symptom scores 